\title{
Structural and Functional Studies of TBEV Non-Structural Protein 5
}

\author{
P. Havlíčková ${ }^{1}$, J. A. Crossley ${ }^{1}$, Z. Gardian ${ }^{1,2}$, I. Kutá Smatanová ${ }^{1}$ and Z. Franta ${ }^{1}$ \\ ${ }^{1}$ Department of Chemistry, Faculty of Science, University of South Bohemia, Branišovská 1760, České Budějovice, Czech Republic \\ ${ }^{2}$ Institute of Parasitology, Biology Center of the Czech Academy of Sciences, České Budějovice, Czech Republic
}

zfranta@prf.jcu.cz

Tick-borne encephalitis virus (TBEV) is a major human pathogen, transmitted by ticks from family Ixodidae. TBEV is an enveloped virus with $\mathrm{a} \sim 11 \mathrm{~kb}$ positive-sense single-strand RNA genome, encoding a single $375 \mathrm{kDa}$ polyprotein. During infection, the polyprotein is cleaved into three structural and seven non-structural (NS) proteins. While the structural proteins are involved in assembly of new virions, the non-structural proteins are responsible for virus replication.

Non-structural protein 5 (NS5) is a large bi-functional conserved protein comprising two domains connected by a highly flexible linker, which is important for the activity as well as determines the overall shape of the protein. N-terminal methyltransferase (MTase) domain is the capping enzyme. The C-terminal RNA-dependent RNA polymerase (RdRp) is crucial for virus replication.

This project aims at structure determination and functional studies of TBEV NS5 protein. Various gene constructs were designed and cloned: NS5 full length, RdRp domain and MTase domain. Expression and purification of individual products have been optimized and pure proteins were used for initial crystallization screening, cryo-EM analysis and functional assays.

So far, we have obtained cryo-EM data for RdRp domain, using Titan Krios equipped with Falcon 4 camera and Relion processing pipeline yielded a reconstruction of $6 \AA$ resolution. Tiny protein crystals of RdRp grew in several crystallization conditions. Furthermore, fluorescence-based binding assays revealed substrate affinity and specificity.

Keywords: RNA-dependent RNA polymerase, non-structural protein 5, tick-borne encephalitis virus

This research is supported by ERDF No. CZ.02.1.01/0.0/0.0/15_003/000041, GAJU 106/2021/P and GAJU 04-17/2019/P. 